Advances in Epilepsy Monitoring by Detection and Analysis of Brain Epileptiform Discharges

Bahman Abdi-Sargezeh and Saeid Sanei

School of Science and Technology, Nottingham Trent University, UK

Author Note

Any correspondence should be referred to Saeid Sanei, School of Science and Technology, Nottingham Trent University, Clifton Campus, Clifton Ln, Clifton, Nottingham NG11 8NS, UK. Email: saeid.sanei@ntu.ac.uk 


\begin{abstract}
Brain interictal and pre-ictal epileptiform discharges (EDs) are transient events occurred between two or before seizure onsets visible in intracranial electroencephalographs. In the diagnosis of epilepsy and localization of seizure sources, both interictal and ictal recordings are extremely informative. For this propose, computerized intelligent spike and seizure detection techniques have been researched and are constantly improving. This is not only to detect more EDs from over the scalp but also to classify epileptic and non-epileptic discharges. Tensor factorization and deep learning are two advanced and powerful techniques which have been recently suggested for ED detection. Here, our main contribution is to review recent ED detection methods with emphasis on multi-way analysis and deep learning approaches. These techniques have opened a new window to the epilepsy diagnosis and management spheres.
\end{abstract}

Keywords: Brain epileptiform discharges, deep learning, EEG, multi-way analysis, tensor factorization. 


\section{Advances in Epilepsy Monitoring by Detection and Analysis of Brain Epileptiform}

\section{Discharges}

Epilepsy is a common neurological condition affecting more than 50 million people globally. It is a chronic brain disease characterized by epileptic seizures occurring due to excessive discharges of a group (or groups) of neurons in the cerebral cortex or hippocampus. The mainstay of diagnosis remains detection of interictal or pre-ictal epileptiform discharges (EDs) which occur between two seizure onsets. ED signatures can be captured using electroencephalography (EEG) (Sanei, 2013).

ED identification can establish a guideline for pre-ictal state monitoring, seizure prediction, treatment and surgical planning. Seizure prediction mitigates taking regular anticonvulsants, preliminary surgical interventions for identification of ED generators, and the hazards of fall injury. In the traditional seizure prediction methods, the increase in synchrony and decrease in the chaotic behavior of cortical activities have been the main quantification parameters, regrettably, with low accuracy and insufficient consistency in terms of seizure types and across the subjects. Now, identification of the EDs from the scalp EEG (sEEG) up to sufficient accuracy has raised hopes for developing a new direction for more concise seizure prediction. The techniques used in this research involve many advanced signal processing techniques and their associated mathematics. Here, recent research in multiway analysis (based on tensor factorization) and deep neural networks (DNNs) for ED detection is reviewed.

In terms of their morphology, EDs can fall into five groups: (1) spike, lasting for 20-70 ms and detectable from the background activity; (2) sharp wave, the same as spikes but lasting for 70-200 ms; (3) sharp-and-slow-wave complex, comprised of a sharp wave followed by a slow wave; (4) spike-andslow-wave complex, consisting of a spike followed by a slow wave; and (5) multiple spike-and-slow-wave complexes, the same as spike-and-slow-wave complex but with two or more spikes associated with one or more slow waves (Kane et al., 2017). In addition, depending on the location of seizure sources, spatial distribution of EDs vary.

There are several challenges in using scalp-recorded data to automatically detecting EDs. The first challenge is that studies conducted on concurrent sEEG and intracranial EEG (iEEG) recordings have revealed that standard electrodes record only a relatively small proportion of spikes detectable from over the scalp or at the cortical surface. Nayak and colleagues recorded simultaneously sEEG (using 20 standard scalp electrodes) and iEEG (using 12 foramen ovale (FO) electrodes) from patient suffering from temporal lobe epilepsy during 1990 to 1998 (Nayak et al. 2004). Their study has shown that only $9 \%$ of EDs are observable over the scalp without averaging across ED segments or concurrent iEEG recording. Less than thirteen percent (12.8\%) of EDs were detectable over the scalp as a small transient by referring to concurrent iEEG as a ground truth, and $59.7 \%$ of EDs were identified over the scalp by referring to simultaneous iEEG and averaging across ED segments. Finally, 18.7\% of 
EDs showed no signal or signature on the scalp. Later, Yamazaki el al. compared dense EEG (dEEG) and iEEG for interictal spike detection (Yamazaki et al., 2012). dEEG was recorded using 256 standard electrodes and, for recording the iEEG, from 48 to 102 subdural strip and grid electrodes were implanted over the mesial and lateral temporal lobe. They showed that $45 \%$ of spikes can be detected over dEEG. However, by reducing the number of channels to 19 according to 10-20 system, they detected only $22 \%$ of spikes over the scalp. Nayak et al. (2004) and Yamazaki et al. (2012) obtained different results in terms of the percentage of visible EDs from over the scalp. This can be due to the recording systems since Nayak et al. (2004) recorded the signal much earlier than Yamazaki et al. (2012). This challenge has been addressed in very few studies (Antoniades et al., 2018, Abdi-Sargezeh et al. $2021 \mathrm{~b}$ c). One way to solve this problem is to record the sEEG and iEEG signals simultaneously, then use iEEG as ground truth for ED annotation and detect EDs from sEEG recordings AbdiSargezeh et al., 2021b c). Another way is to derive iEEG from sEEG signals. Antoniades et al. (2018) employed DNN algorithms to map sEEG to iEEG recordings (in other words, estimated iEEG from sEEG recordings).

The second challenge in automatically detecting EDs is that they are similar to many normal brain activities and artifacts such as extracerebral potentials from muscles, eyes, heart, and electrodes. Eye blink and heart beat artifacts also resemble spike waves. However, they differ from EDs in terms of source location and frequency range. Therefore, analysis of EEG signals in frequency domain and involving space diversity can suppress such artifacts. Through multi-way analysis, spatial and frequency components were analyzed and used for detecting epileptic spikes (Thanh et al., 2020).

The final challenge in ED detection is uncertainty in annotating a waveform as ED. There is a huge disagreement among expert epileptologists as to whether a wave is an ED or not. Webber and colleagues conducted a research to find out how electroencephalographers (EEGers) interpret EEG signals (Webber et al., 1993). Eight EEGers scored EDs in twelve short EEG records. Only $18 \%$ were marked as EDs by all the EEGers and 38\% by only one EEGer. Quite recently, Halford performed a research to evaluate the neurologist performances in ED detection (Halford et al., 2018). Thirtyfive EEGers participated in the research and were supposed to annotate the EDs in 200 EEG segments each with the length of 300 seconds. The number of events marked as EDs was different among EEGers from 6 to 212 (with the mean of 67.7). Quite recently, in an effective approach, for the first time the uncertainty in labelling the EDs has been incorporated in an ED detection system via multiway analysis (Abdi-Sargezeh et al., 2021b).

Here, at first, ED datasets and performance metrics are described; then, the traditional methods employed for ED detection are briefly reviewed. Finally, we further elaborate on our recent contributions in the field which are application of multi-way analysis and DNNs in ED detection. 


\section{ED Data}

There is no public scalp ED dataset and each research group has used their own private dataset for detection of EDs from over the scalp. However, an intracranial ED dataset (Janca et al., 2015) is publicly available at https://isarg.fel.cvut.cz/downloads/spike-detector/. Table 1 summarizes the datasets used in some previous studies. Here, the most used private ED dataset and the public intracranial ED dataset are described in detail.

A research group led by prof. Saeid Sanei has been working on concurrent scalp and intracranial recordings (Spyrou et al., 2015, 2016, 2018, Antoniades et al., 2016, 2017, 2018, Abdi-Sargezeh et al. $2021 \mathrm{~b} \mathrm{c}$. sEEG and iEEG signals were simultaneously recorded at the sampling rate of $200 \mathrm{~Hz}$ at the Department of Clinical Neurophysiology at the Maudsley and Kings College Hospitals. Eighteen (18) standard silver chloride electrodes and 12 FO electrodes (Wieser et al., 1985) were respectively used for recording sEEG and iEEG signals from 25 patients suffering from mesial temporal lope epilepsy. Scalp electrodes were implemented according to the 'Maudsley' electrode placement system (Torre et al. 1999 , Nayak et al., 2004). The Maudsley system is superior to the standard 10-20 system in capturing EDs signatures from those suffering from seizures arising from mesial temporal structures. This system is essentially similar to $10-20$ system (the electrodes have the same name) except that mid-temporal, posterior-temporal and occipital electrodes in the Maudsley system are approximately $20 \mathrm{~mm}$ lower than that in the 10-20 system. Therefore, it provides more extensive coverage of the lower part of the cerebral convexity and adapts itself to cranial asymmetries (Binnie et al. 1982), increasing the sensitivity for recording from basal sub-temporal structures. In terms of intracranial recordings, FO electrodes are introduced through the infraorbital foramen and directly placed on the exposed mesial temporal structures. Thus they not only enable capturing ED signatures with extremely high sensitivity but also provide a unique opportunity to simultaneously record sEEG and iEEG without disrupting the brain coverings. Figure 1 shows the lateral and basal X-radiographs of scalp and intracranial FO electrodes.

The public intracranial ED dataset (https://isarg.fel.cvut.cz/downloads/spike-detector/) consists of recordings from seven patients with refractory epilepsy who underwent invasive exploration as a part of the presurgical examination. The number of electrodes was different among subjects (the median number of electrodes was 65). However, to avoid human labelling bias, only 15 electrodes with the highest spike rate were selected from each dataset. Five-minute iEEG recordings of each subject were analyzed and made publicly available. Three neurophysiologists with at least 10-year experience reviewed the data and labelled the spikes. Each spike was labeled as either obvious or ambiguous. Waveforms associated with any kind of hesitation and/or doubts were classified as ambiguous. Obvious and ambiguous spikes on which two or more readers agreed were selected as gold standard spikes (GS). Table 2 presents the patient characteristics (sex, age, number of GS, epilepsy location, seizure 
duration, and pathology) for each of the seven patient.

\section{Performance Metrics}

To evaluate the performance of an ED detection system, different metrics have been utilized. They are accuracy (ACC), sensitivity (SEN), specificity (SPC), false positive per minute (FP/min), and area under the receiver operating characteristic curve (AUC). Accuracy presents how accurately the EDs and non-EDs are detected. SEN shows the ability of a system in correctly detecting EDs. SPC shows the ability of a system in correctly detecting non-EDs. FP/min illustrates the number of non-ED segments recognized as ED segments in a minute. AUC measures the entire two-dimensional area underneath the entire receiver operating characteristic (ROC) curve, which is a probability curve showing the performance of a classifier for different thresholds. ROC curve is plotted with SEN against (1-SPC) where SEN is on the y-axis and (1-SPC) is on the $\mathrm{x}$-axis.

ACC, SEN, and SPC are defined as follows:

$$
\begin{aligned}
\mathrm{ACC} & =\frac{T P+T N}{T P+F P+T N+F N} \times 100 \%, \\
\mathrm{SEN} & =\frac{T P}{T P+F N} \times 100 \%, \\
\mathrm{SPC} & =\frac{T N}{T N+F P} \times 100 \%,
\end{aligned}
$$

where TP is the number of ED samples classified correctly in the ED class, TN represents the number of non-ED samples recognized accurately as non-ED samples, FP indicates the number of non-ED samples detected incorrectly as ED samples, and FN indicates the number of ED samples categorized wrongly in the non-ED class.

\section{Traditional Methods in ED Detection}

Whilst several methods and approaches have been developed for automatically detecting EDs, the current review presents some of the most popular approaches.

\section{Component Analysis}

Principal component analysis which exploits uncorrelated components was employed to the decorrelation of epileptic and normal EEGs (Wang et al., 2010). Independent component analysis (ICA) as a powerful tool in signal processing has been widely applied to ED detection (Kobayashi et al. 2001, 1999, Hesse and James, 2006). Lucia and colleagues developed a model based on ICA to discriminate the epileptiform activities from eye blink artifacts (De Lucia et al., 2008). During the last decade, epilepsy researchers have paid much attention to sparse representation (Yuan et al., 2014, 
2016). A sparse representation of data is a representation in which only few samples in a signal segment are not zero. The train of spikes emitted from individual neurons in the brain can be considered sparse in some domains such as time and space domains. One of the interesting characteristics of EDs is their sparsity in the time domain. Quite recently, a model was proposed to detect epileptic EEG spikes based on sparse representation (Jiang et al., 2020).

\section{Template Matching}

In template matching method, an ED template is manually selected from EEG recordings and a waveform is recognised as ED whenever its cross-correlation with the template exceeds the predefined treshold value. Since 1972, when the template matching was used for the first time for seizure detection (Stevens et al. 1972), this method has been broadly used for ED detection (Lodder et al. 2013; Lodder and van Putten, 2014, Thomas et al., 2017).

\section{Feature Representations}

In addition to detection of EDs from a single channel, temporal and spatial information are of paramount importance to neurologists in identifying a waveform as an ED. In feature representation methods, some features are extracted and used for ED detection.

\section{Mimetic Technique}

In this approach, the necessary features used by neurologists for identifying EDs are extracted. The distinctive characteristics of EDs such as slope, height, duration, and sharpness are compared with the benchmark values presented by the neurologists (Tzallas et al., 2012). The most popular method in the mimetic technique category is to decompose the EEG waveforms into a couple of halfwaves and extract the mimetic features from them (Gotman and Gloor, 1976, Faure, 1985, Webber et al. 1994, Wang et al., 2020). Quite recently, a multi-step ED detection algorithm was proposed (Wang et al. 2020). In the feature extraction step, the authors decomposed EDs into two half-waves and extracted some waveform features (i.e., the amplitude, duration and slope of left and right halfwaves of spike, etc). The major drawback of this particular method is that it ignores the ED spike shape variations across subjects, ages, and even trials.

\section{Time-frequency Representation}

Effectiveness of time-frequency representation in EEG signal processing has been shown. Timefrequency representation separates the main components of EDs from the background activity. Wavelet (Sartoretto and Ermani, 1999, Göksu, 2018, Indiradevi et al., 2008), Fourier (Polat and Güneş, 2007), and Hilbert transforms (Zhu et al. 2015) have been investigated in ED literature. 
A large proportion of EDs are invisible over the scalp (Nayak et al., 2004, Yamazaki et al., 2012). In an effective approach, Spyrou and colleagues detected EDs from the concurrent sEEG using time-frequency features (Spyrou et al., 2016). The sEEG and iEEG signals were simultaneously recorded. The iEEG recordings were used as ground truth for ED annotation by an expert EEGer. The authors employed the spectrogram method (which is a way of representing the signal strength over time at various frequencies) to extract time-frequency features from the sEEG. Finally, both scalpvisible and scalp-invisible EDs were detected from over the scalp.

\section{Classification of Nonlinear Features}

Nonlinear features such as correlation dimension and largest Lyapunov exponent have been used in ED and seizure detection (Corsini et al. 2006, Iasemidis et al., 1990, Gajic et al., 2015). Gajic and colleagues developed a method based on time-frequency and nonlinear features to detect EDs (Gajic et al., 2015). Not only some features from time, frequency, and time-frequency domains were extracted but also the correlation dimension and the largest Lyapunov exponent were obtained as nonlinear features to boost the robustness and performance of the model.

\section{Advanced Techniques in ED Detection}

These days, computers have superb computational power, which enables data scientists particularly neuro-engineering researchers to tackle challenges that once were hard to achieve. Therefore, multi-way analysis and DNNs requiring high computational complexity have recently attracted the attention of scientists and researchers. Multi-way analysis (i.e., tensor factorization) makes the data diversity is taken into account towards achieving efficiency and optimality. It can effectively exploit the data variations in different domains such as time, frequency, space, subject, and trial all together in their formulation (Abdi-Sargezeh et al. 2021a; Spyrou et al., 2015). Furthermore, DNNs exploit the significance of each individual data sample in learning features which wouldn't be achieved through the use of "traditional classifiers" (i.e., those classifiers - such as support vector machines, linear discriminant analysis, and decision tree - that only map input features to a category and are unable to learn the features from the input data). Here, the aim of this paper is to review the recent literature in ED detection that enjoyed tensor factorization or DNNs in designing their models.

\section{Multi-way Analysis}

Mathematically, matrix is a two-dimensional array while tensor is multidimensional which can best accommodate the diversity (e.g. time, frequency, space, trial, and subject) in the data. Table 3 summarizes studies employing multi-way analysis for ED and epileptic detection. Type of recording 
(sEEG or iEEG), tensorization method, decomposition method, the used features for classification, and the performance of methods are illustrated.

There are different methods for decomposing a tensor (Kolda and Bader, 2009). The most popular methods which have been employed for ED detection are CANDECOMP/PARAFAC decomposition (CPD) (Abdi-Sargezeh et al., 2021b) and Tucker decomposition (TD) (Spyrou et al., 2015).

CPD decomposes a tensor into the sum of rank-one components. Suppose we are given a threeway tensor $\mathcal{X} \in \mathbb{R}^{L \times M \times N}$. According to CPD problem, the tensor $\mathcal{X}$ can be formulated as:

$$
\mathcal{X} \approx \sum_{r=1}^{R} \mathbf{a}_{r} \circ \mathbf{b}_{r} \circ \mathbf{c}_{r}
$$

where the symbol 'o' represents the vector outer product, $R$ is a positive integer and $a_{r} \in \mathbb{R}^{L}, b_{r} \in$ $\mathbb{R}^{M}$, and $c_{r} \in \mathbb{R}^{N}$ for $r=1, \ldots, R$. This is illustrated in Figure 2 .

The factor matrices are constructed from the combination of the rank-one tensors, i.e., $\mathbf{A}=$ $\left[\mathbf{a}_{1} \ldots \mathbf{a}_{R}\right]$. Following the "Kruskal operator" (Kolda, 2006), (1) can be modified to

$$
\mathcal{X} \approx \llbracket \mathbf{A}, \mathbf{B}, \mathbf{C} \rrbracket \equiv \sum_{r=1}^{R} \mathbf{a}_{r} \circ \mathbf{b}_{r} \circ \mathbf{c}_{r} .
$$

where $\mathbf{A} \in \mathbb{R}^{L \times R}$ and $\mathbf{B} \in \mathbb{R}^{M \times R}$, and $\mathbf{C} \in \mathbb{R}^{N \times R}$ are factor matrices. The problem of computing CPD (2) can be formulated as a least-square optimization problem:

$$
\min _{\mathbf{A}, \mathbf{B}, \mathbf{C}} f \equiv \frac{1}{2}\|\mathcal{X}-\llbracket \mathbf{A}, \mathbf{B}, \mathbf{C} \rrbracket\|^{2} .
$$

On the other hand, TD decomposes a tensor into a core tensor multiplied by a matrix along each mode. In the three-way case where $\mathcal{X} \in \mathbb{R}^{L \times M \times N}$, the aim is to find a tensor $\overline{\mathcal{X}} \in \mathbb{R}^{L \times M \times N}$, having $\operatorname{rank}_{1}(\overline{\mathcal{X}})=P, \operatorname{rank}_{2}(\overline{\mathcal{X}})=Q$, and $\operatorname{rank}_{3}(\overline{\mathcal{X}})=R$, that minimizes the least-squares cost function

$$
j(\overline{\mathcal{X}})=\|\mathcal{X}-\overline{\mathcal{X}}\|^{2}
$$

The tensor $\overline{\mathcal{X}}$ can be decomposed to

$$
\overline{\mathcal{X}}=\mathcal{G} \times{ }_{1} \overline{\mathbf{A}} \times{ }_{2} \overline{\mathbf{B}} \times{ }_{3} \overline{\mathbf{C}},
$$

where $\overline{\mathbf{A}} \in \mathbb{R}^{L \times P}, \overline{\mathbf{B}} \in \mathbb{R}^{M \times Q}$, and $\overline{\mathbf{C}} \in \mathbb{R}^{N \times R}$, all with orthonormal columns, are factor matrices. $\mathcal{G} \in$ $\mathbb{R}^{P \times Q \times R}$ is the core tensor and its entries present the level of interaction between the factor matrices. Note that $\times_{i}$ shows the $i$-th mode product. The reader is referred to De Lathauwer et al. (2000) for more details. The schematic of TD is shown in Figure 3 
After decomposition, the core tensor and factor matrices can be either individually used for classification or projected onto the main data for classification. For instance, in Abdi-Sargezeh et al. (2021b), the authors concatenated ED segments $\mathbf{X}_{n} \in \mathbb{R}^{L \times M}, n=1, \ldots, N$, into a three-way tensor $\mathcal{X} \in \mathbb{R}^{L \times M \times N}$ - where $L, M$, and $N$ are the number of time samples, EEG channels, and ED segments. After decomposing the tensor into factor matrices via CPD (3) and obtaining the temporal $\mathbf{A} \in \mathbb{R}^{L \times R}$, spatial $\mathbf{B} \in \mathbb{R}^{M \times R}$, and segmental $\mathbf{C} \in \mathbb{R}^{N \times R}$ factors, the data is projected onto the spatial factors, $\mathbf{Y}_{k}=\mathbf{X}_{k} \mathbf{B}$, where $\mathbf{X}_{k} \in \mathbb{R}^{L \times M}(k=1, \ldots, K)$ is an ED or non-ED segment from the training or test data and $\mathbf{Y}_{k} \in \mathbb{R}^{L \times R}(k=1, \ldots, K)$ is the projected ED or non-ED segment used for classification. The schematic of the proposed method in Abdi-Sargezeh et al. (2021b) is illustrated in Figure 4

Much research has been undertaken recently for detection of epileptic seizure through multiway analysis (Hunyadi et al., 2017; Acar et al., 2007b; Ontivero-Ortega et al., 2015, Aldana et al. 2018: Yuan et al. 2020). Acar et al. (2007b) employed continuous wavelet transform (CWT) - which is an efficient way of presenting signal variations in both time and frequency using some well-known basis waveforms called wavelet - using Mexican-hat wavelet (a low-oscillation wavelet) as the mother wavelet to obtain the time-frequency features. Then, a three-way tensor with the dimension of time, scale, and channel was constructed. Finally, N-way partial least squares (N-PLS) was employed to extract features in multi-dimensions. Aldana and colleagues employed CPD and BTD to detect nonconvulsive epileptic seizure (Aldana et al., 2018). To expand EEG recordings into a tensor, the authors decomposed EEG recordings using CWT or Hilbert-Huang transform (HHT) and construct a threeway tensor with the dimension of frequency $\times$ time $\times$ channel. They followed four configurations; CWT-CPD, CWT-BTD, HHT-CPD, and HHT-BTD. Overall, HHT outperformed CWT. The best performance was obtained using HHT-CPD through the spatial features, closely followed by HHTBTD through the frequency features. There was no significant difference between two types of tensor factorization methods used in their research. They achieved different performances depending on the feature space. CPD outperformed when the spatial features were used and BTD outperformed when the frequency features were applied for the classifications. In Yuan et al. (2020), the authors employed modified Stockwell transform to obtain frequency features. TD was applied to decompose the tensor into its factors and the core tensor. Finally, the core tensor was vectorized and used as the EEG features for seizure detection.

In addition to employing tensor-based methods in epileptic seizure detection, tensor decomposition has been applied for seizure source localization. Acar et al. (2007a) and De Vos et al. (2007) employed CPD for localizing seizure sources. Hunyadi and colleagues introduced BTD to detect the seizure source zone (Hunyadi et al., 2014). The authors compared their proposed BTD-based method with a CPD-based model. BTD-based method localized seizure sources better than CPD-based method. 
In Hunyadi et al. (2014), each EEG channel was normalized using z-score normalization method. However, generally, the normalization of channel separately affects the spatial and temporal information and consequently the localization process fails. Therefore, z-score normalization of each channel and also employing common average reference as an artifact removal method can cause error in tensorbased EEG analysis.

Tensor decomposition was applied for ED detection for the first time in Spyrou et al. (2015). The authors employed their proposed model to detect EDs from iEEG. The spectrogram method was applied to obtain time-frequency features. Then, they constructed a four-way tensor with the dimension of channel, time, frequency, and segment (illustrated in Figure 5) employed TD to decompose the tensor into spatial, temporal and spectral factors. Finally, spatial factors were used as features for ED detection. Later, the same authors employed TD to detect EDs from sEEG (Spyrou et al., 2018). They detected EDs in two different approaches, namely within-subject and leave-one-subject-out approaches. In addition, different feature spaces (the core tensor and spatial factors in the within-subject approach and temporal and spatial features in the leave-one-subject-out approach) were used in ED detection. Quite recently, Thanh et al. (2020) introduced nonnegative TD (NTD) with a novel tensor formulation to detect the spikes. At first, the authors decomposed the EEG channels using CWT. Then, they constructed a four-way tensor by incorporating channel, time, frequency, and spike segment. The tensor decomposed using NTD to obtain the factor matrices and the core tensor. Finally, both spike and non-spike segments from the training and test datasets were projected onto the factor matrices to extract the features for classification. The main differences between Spyrou et al. (2015, 2018) and Thanh et al. (2020) algorithms are: (1) NTD was used in Thanh et al. (2020) while TD in Spyrou et al. (2015, 2018) and (2) only epileptic spikes were concatenated to a forth order tensor in Thanh et al. (2020) while in Spyrou et al. (2015, 2018) all ED and non-ED segments were utilized. Since different datasets were used in the mentioned papers, making a comparison between the two methods is difficult. In Thanh et al. (2020), only the sEEG was recorded and analyzed, meaning that only scalp-visible spikes were detected. Meanwhile, sEEG and iEEG were simultaneously recorded in Spyrou et al. (2018) and all scalp-visible and scalp-invisible EDs were detected from the concurrent sEEG.

Uncertainty in labelling biomedical signals and images is a crucial factor which may seriously affect the diagnosis (Abdi-Sargezeh et al., 2021b c). In the case of seizure, the EDs are similar to some normal brain activities or artifacts (e.g., eye blink artifacts). In Abdi-Sargezeh et al. (2021b), a tenorbased model has been developed to incorporate the probability of a waveform being an ED in an automatic ED detection system. An expert epileptologist provides a score showing his confidence level in labeling each ED. Figure 7 shows the EDs with different scores. Concurrent iEEG (channels R1 to R6 and L1 to L6) and sEEG recordings have been analyzed to detect both scalp-visible and scalp-invisible 
EDs. From Figure 7, there is no sign of epileptic spikes in EDs scored 1 to 3 in the scalp channels. In the EDs scored 4 and 5, there are epileptic spikes in both intracranial and scalp channels. For automatically detecting EDs, the authors have constructed a three-way data tensor of time samples, channels, and ED segments and a three-way probability tensor based on the EDs scores. Then, a CPD weighted optimization algorithm (Acar et al. 2011) effectively exploits the uncertainty levels and decomposes the data and probability tensors to achieve the related tensor factors. Finally, both EDs and non-EDs are projected into the spatial factors to achieve the discriminative features.

\section{Deep Neural Networks}

Shallow neural networks (SNNs) have been broadly used in ED detection from the appearance of neural networks. Some researchers used raw EEG signals for ED detection (Eberhart et al., 1989 Özdamar and Kalayci, 1998), others extracted some features (mimetic features, time-frequency representation, etc.) from ED or spike segments and applied them to an SNN to be classified (Gabor and Seyal, 1992; Argoud et al., 2006, Tzallas et al., 2006). However, SNNs are inefficient when the dataset is noisy.

DNNs, as a state-of-the-art method in artificial intelligence, have been used in data science particularly for biomedical signal processing (Procházka et al., 2020, Craik et al., 2019, Jarchi et al. 2020) and ED detection. The studies using DNN algorithms for ED detection are summarized in Table 4. Among DNN algorithms, convolutional neural networks (CNNs) are the most popular ones.

CNNs are mainly comprised of three types of layers: convolutional layers, pooling layers and fully-connected layers. A CNN architecture is formed by stacking these layers. A simplified CNN architecture is shown in Figure 6. The convolution layer applies a convolution to the previous layer data and forwards the result to the next layer. It is followed by a non-linear activation function. The pooling layer simply performs downsampling along the spatial dimensionality of the given input, whereby the number of parameters is reduced within that activation. Finally, the fully-connected layer performs like standard neural networks.

Antoniades et al. (2016) and Johansen et al. (2016) introduced DNNs to detect EDs from EEG for the first time, though DNNs were already employed to predict epileptic seizures (Mirowski et al. 2008). Two CNNs with different topologies were trained for detecting EDs from iEEG by Antoniades et al. (2016). On the other hand, CNN was employed to detect EDs from sEEG by Johansen et al. (2016). Since the mentioned studies evaluated their proposed models via different recordings, making a comparison between them is difficult. In another study, EDs were given a score according to their morphology as well as their spatial information captured through multichannel recording, and a CNN was employed to detect EDs with different scores from iEEG (Antoniades et al., 2017) by developing a multiclass ED detection model. Abou Jaoude et al. (2020) developed a CNN-based method 
to detect the mesial temporal lobe EDs from a single bipolar intracranial channel. The authors averaged the CNN outputs of multiple bipolar channels to determine whether the test segment is related to an ED event or not. Thomas and colleagues detected the scalp EDs using a 1D CNN through a single channel approach (Thomas et al., 2020). The EDs were labeled separately for each channel and 1D CNN was trained to detect EDs from a single channel. Finally, the channel outputs were combined to produce a single value used as an index to identify an ED segment. They evaluated their proposed method in three different ED datasets.

Recording sEEG and iEEG simultaneously provides an opportunity to detect the scalp-visible and scalp-invisible EDs from over the scalp. Furthermore, mapping sEEG to iEEG ameliorates the model performance. This approach significantly outperformed other compared methods. In a promising approach, the researchers estimated the intracranial samples using scalp recordings via DNNs $(\overline{A n-}$ toniades et al. 2018). A concurrent sEEG and iEEG recordings were analyzed. The authors developed an asymmetric-symmetric autoencoder (ASAE) to map sEEG to iEEG. Then, a CNN was applied to the mapped signal to detect EDs. Figure 8 shows the topology of ASAE. An autoencoder (AE) requires a weigh matrix since it is symmetric and the same weight is employed for encoding and decoding. The developed asymmetric $\mathrm{AE}(\mathrm{AAE})$ requires two weight matrices with different dimensions, $\mathbf{W}^{e}$ and $\mathbf{W}^{d}$ for encoding and decoding operations respectively. For mapping sEEG into iEEG, the developed AAE was followed by an AE to enhance the estimated pseudo-iEEG. The estimated signals are shows in Figure 9. A leave-one-subject-out approach was used for ED detection. The iEEG recordings of training subjects were only used in training the model. In other words, only the sEEG recording of test subject was used to detect the EDs. The model achieved $68 \%$ accuracy. The same dataset was used in Spyrou et al. (2016), in which only sEEG signals were employed to detect EDs. The accuracy of $65 \%$ was achieved, $3 \%$ less than the accuracy obtained in Antoniades et al. (2018).

In Tjepkema-Cloostermans et al. (2018), LSTM and CNN networks were applied to detect focal EDs. Five different architectures, namely 1D CNN, 2D CNN, LSTM, combined 1D CNN and LSTM, and combined 2D CNN and LSTM, were implemented. In 1D CNN, the filters were defined for each channel separately, while, in 2D CNN, the filters were two-dimensional involving temporal and spatial information. 2D CNN outperforms 1D ones. As a consequence, the combination of temporal and spatial information in the convolutional layers may boost the model performance. However, the model did not provide a good sensitivity although the authors trained 346 different neural networks to achieve the best result. They used 2-second ED segments which is unnecessarily long. The length of ED segments is usually selected to be less than $500 \mathrm{~ms}$ in most of ED detection studies (Spyrou et al. 2016: Thanh et al. 2020; Spyrou and Sanei, 2016) since the length of an ED waveform is not longer than $200 \mathrm{~ms}$ (Halford et al. 2013). Therefore, using too long ED segments leads the model to fit over non-ED segments. 
Prasanth and colleagues applied frequency sub-bands - Delta (1 to $4 \mathrm{~Hz}$ ), Theta (4 to $8 \mathrm{~Hz})$, Alpha (8 to $13 \mathrm{~Hz}$ ), and Beta (13 to $30 \mathrm{~Hz}$ ) - and the raw EEG as input to a DNN model to detect EDs (Prasanth et al., 2020). They concatenated frequency sub-bands in two ways: (1) by concatenation of frequency sub-bands horizontally into a long vector, i.e., the dimension of new feature vector was $\mathbb{R}^{1 \times(L \times N)}$, where $L$ and $N$ are respectively the number of time samples and sub-bands, and (2) the concatenation of frequency sub-bands vertically into a matrix with the dimension of $\mathbb{R}^{L \times N}$. $1 \mathrm{D}$ CNN was used in the first implementation and 2D CNN in the second one. 1D CNN outperformed 2D CNN, meaning that the model performance was deteriorated when the information from different sub-bands in the convolution layers were combined. Most of the ED information lie in the high frequency bands. Therefore, by dividing the EEG into sub-bands, the ED morphology changes and consequently the model performance deteriorates. The obtained results of each sub-band in Prasanth et al. (2020) proves this issue. The best false positive rate per minute (FP rate/min) provided via sub-bands was 85 while the raw EEG provided $0.36 \mathrm{FP}$ rate/min. Though combined all sub-bands and raw EEG achieved higher FP rate/min 0.23 compared to only raw EEG, the result of combination of only subbands were not reported, which may be significantly worse than that achieved by using raw EEG. Furthermore, the minimum length of spikes is $20 \mathrm{~ms}$ and can be as long as $50 \mathrm{~Hz}$. Meanwhile, the Gamma band $(>30 \mathrm{~Hz})$ was completely suppressed in Prasanth et al. (2020) which is likely to have significant information regarding the spikes particularly in iEEG recordings (Medvedev, 2001). Due to this fact, in some studies only Gamma band has been used for ED detection (Bourien et al., 2004). However, Gamma band has been suppressed in few ED detection studies (Fukumori et al., 2019). Xuyen and colleagues divided the EEG signals into frequency sub-bands as well (Le et al., 2018). However, they extracted Gamma band $(30-50 \mathrm{~Hz})$ together with other sub-bands. They employed CWT to extract the time-frequency features and deep belief network (DBN) to detect EDs.

To detect epileptic spikes, Fukumori et al. (2020) developed a CNN model based on a bank of linear-phase finite impulse response filters, performing as bandpass filters that extract biomarkers of EDs without destroying waveforms because of linear-phase condition. Quite recently, an LSTM-based model with self-attention was proposed to detect epileptic spikes that were not temporally aligned (Fukumori et al. 2021). The hypothesis of authors was that a neural network embeding a temporal self-attention mechanism enables extraction of the locations and ranges of particular interest within an EEG segment for epileptic spike detection.

\section{Conclusion}

Detection of EDs plays a crucial role in epilepsy diagnosis and management. The development of powerful computers and effective algorithms pave the way to detect more EDs from over the scalp. Tensor factorization and DNNs have attracted researchers in both bioengineering and neuroscience. 
This is mainly because the diversity in the data is best exploited by the use of multi-way systems. Here, the application of multi-way analysis (i.e., tensor factorization) and DNNs are reviewed in detail. Among tensor factorization methods, TD-based methods are more popular for ED detection and CPD algorithm have been commonly employed to detect seizure and localize the seizure sources. Among DNN algorithms, CNN and LSTM are the most popular methods employed for ED detection. Though detecting the EDs particularly from over the scalp is extremely challenging due to the similarity between the EDs and many brain activities and artifacts such as eye blink, the development of tensor factorization and DNNs lead the detection models to be more precise. Convolutional LSTM (Shi et al. 2015) and convolutional tensor-train LSTM (Su et al. 2020) have been recently developed to capture spatio-temporal features of a sequential data. By deploying these algorithms to detect EDs in which the spatio-temporal information is of paramount importance, computer-aided epilepsy diagnostic systems may progress more than ever.

Additionally, more work needs to be done to distinguish between seizure related EDs and the EDs with non-epileptic origin. These non-seizure related EDs include but not limited to sleep spindles and those spikes related to tumors, infarct, and encephalitis. This requires more intelligent systems which glue the advances in both signal processing and machine learning.

\section{Refferences}

Abdi-Sargezeh, B., Foodeh, R., Shalchyan, V., and Daliri, M. R. (2021a). EEG artifact rejection by extracting spatial and spatio-spectral common components. Journal of Neuroscience Methods, 358:109182.

Abdi-Sargezeh, B., Valentin, A., Alarcon, G., and Sanei, S. (2021b). Incorporating uncertainty in data labeling into automatic detection of interictal epileptiform discharges from concurrent scalpEEG via multi-way analysis. International Journal of Neural Systems, page 2150019, ISSN: 0129-0657, https://doi.org/10.1142/S0129065721500192.

Abdi-Sargezeh, B., Valentin, A., Alarcon, G., and Sanei, S. (2021c). Incorporating uncertainty in data labeling into detection of brain interictal epileptiform discharges from EEG using weighted optimization. In ICASSP 2021 - 2021 IEEE International Conference on Acoustics, Speech and Signal Processing (ICASSP), pages 1000-1004. DOI: 10.1109/ICASSP39728.2021.9414463.

Abou Jaoude, M., Jing, J., Sun, H., Jacobs, C. S., Pellerin, K. R., Westover, M. B., Cash, S. S., and Lam, A. D. (2020). Detection of mesial temporal lobe epileptiform discharges on intracranial electrodes using deep learning. Clinical Neurophysiology, 131(1):133-141.

Acar, E., Aykut-Bingol, C., Bingol, H., Bro, R., and Yener, B. (2007a). Multiway analysis of epilepsy tensors. Bioinformatics, 23(13):i10-i18. 
Acar, E., Bingol, C. A., Bingol, H., Bro, R., and Yener, B. (2007b). Seizure recognition on epilepsy feature tensor. In 2007 29th Annual International Conference of the IEEE Engineering in Medicine and Biology Society, pages 4273-4276. IEEE.

Acar, E., Dunlavy, D. M., Kolda, T. G., and Mørup, M. (2011). Scalable tensor factorizations for incomplete data. Chemometrics and Intelligent Laboratory Systems, 106(1):41-56.

Aldana, Y. R., Hunyadi, B., Reyes, E. J. M., Rodriguez, V. R., and Van Huffel, S. (2018). Nonconvulsive epileptic seizure detection in scalp EEG using multiway data analysis. IEEE Journal of Biomedical and Health Informatics, 23(2):660-671.

Antoniades, A., Spyrou, L., Martin-Lopez, D., Valentin, A., Alarcon, G., Sanei, S., and Took, C. C. (2017). Detection of interictal discharges with convolutional neural networks using discrete ordered multichannel intracranial EEG. IEEE Transactions on Neural Systems and Rehabilitation Engineering, 25(12):2285-2294.

Antoniades, A., Spyrou, L., Martin-Lopez, D., Valentin, A., Alarcon, G., Sanei, S., and Took, C. C. (2018). Deep neural architectures for mapping scalp to intracranial EEG. International Journal of Neural Systems, 28(08):1850009.

Antoniades, A., Spyrou, L., Took, C. C., and Sanei, S. (2016). Deep learning for epileptic intracranial EEG data. In 2016 IEEE 26th International Workshop on Machine Learning for Signal Processing (MLSP), pages 1-6. IEEE.

Argoud, F. I., De Azevedo, F. M., Neto, J. M., and Grillo, E. (2006). SADE 3: An effective system for automated detection of epileptiform events in long-term EEG based on context information. Medical and Biological Engineering and Computing, 44(6):459-470.

Binnie, C., Dekker, E., Smit, A., and Van der Linden, G. (1982). Practical considerations in the positioning of EEG electrodes. Electroencephalography and Clinical Neurophysiology, 53(4):453458.

Bourien, J., Bellanger, J.-J., Bartolomei, F., Chauvel, P., and Wendling, F. (2004). Mining reproducible activation patterns in epileptic intracerebral EEG signals: Application to interictal activity. IEEE Transactions on Biomedical Engineering, 51(2):304-315.

Corsini, J., Shoker, L., Sanei, S., and Alarcón, G. (2006). Epileptic seizure predictability from scalp EEG incorporating constrained blind source separation. IEEE Transactions on Biomedical Engineering, 53(5):790-799.

Craik, A., He, Y., and Contreras-Vidal, J. L. (2019). Deep learning for electroencephalogram (EEG) classification tasks: A review. Journal of Neural Engineering, 16(3):031001. 
De Lathauwer, L., De Moor, B., and Vandewalle, J. (2000). On the best rank-1 and rank-(r 1, r 2,..., rn) approximation of higher-order tensors. SIAM Journal on Matrix Analysis and Applications, 21(4):1324-1342.

De Lucia, M., Fritschy, J., Dayan, P., and Holder, D. S. (2008). A novel method for automated classification of epileptiform activity in the human electroencephalogram-based on independent component analysis. Medical \& Biological Engineering \&3 Computing, 46(3):263-272.

De Vos, M., Vergult, A., De Lathauwer, L., De Clercq, W., Van Huffel, S., Dupont, P., Palmini, A., and Van Paesschen, W. (2007). Canonical decomposition of ictal scalp eeg reliably detects the seizure onset zone. NeuroImage, 37(3):844-854.

Eberhart, R., Dobbins, R., and Webber, W. (1989). EEG waveform analysis using CaseNet. In Images of the Twenty-First Century. Proceedings of the Annual International Engineering in Medicine and Biology Society, pages 2046-2047. IEEE.

Faure, C. (1985). Attributed strings for recognition of epileptic transients in EEG. International Journal of Bio-Medical Computing, 16(3-4):217-229.

Fukumori, K., Nguyen, H. T. T., Yoshida, N., and Tanaka, T. (2019). Fully data-driven convolutional filters with deep learning models for epileptic spike detection. In ICASSP 2019-2019 IEEE International Conference on Acoustics, Speech and Signal Processing (ICASSP), pages 27722776. IEEE.

Fukumori, K., Yoshida, N., Sugano, H., Nakajima, M., and Tanaka, T. (2020). Epileptic spike detection by using a linear-phase convolutional neural network. DOI: 10.1101/2020.10.08.330936.

Fukumori, K., Yoshida, N., and Tanaka, T. (2021). Epileptic spike detection by recurrent neural networks with self-attention mechanism. bioRxiv, DOI: 10.1101/2021.06.17.448793.

Fürbass, F., Kural, M. A., Gritsch, G., Hartmann, M., Kluge, T., and Beniczky, S. (2020). An artificial intelligence-based EEG algorithm for detection of epileptiform EEG discharges: Validation against the diagnostic gold standard. Clinical Neurophysiology, 131(6):1174-1179.

Gabor, A. J. and Seyal, M. (1992). Automated interictal EEG spike detection using artificial neural networks. Electroencephalography and clinical Neurophysiology, 83(5):271-280.

Gajic, D., Djurovic, Z., Gligorijevic, J., Di Gennaro, S., and Savic-Gajic, I. (2015). Detection of epileptiform activity in EEG signals based on time-frequency and non-linear analysis. Frontiers in Computational Neuroscience, 9:38, ISSN: 1662-5188. 
Geng, D., Alkhachroum, A., Bicchi, M. A. M., Jagid, J. R., Cajigas, I., and Chen, Z. S. (2021). Deep learning for robust detection of interictal epileptiform discharges. Journal of Neural Engineering, 18(5):056015, https://doi.org/10.1088/1741-2552/abf28e.

Gotman, J. and Gloor, P. (1976). Automatic recognition and quantification of interictal epileptic activity in the human scalp EEG. Electroencephalography and Clinical Neurophysiology, 41(5):513529 .

Göksu, H. (2018). EEG based epileptiform pattern recognition inside and outside the seizure states. Biomedical Signal Processing and Control, 43:204 - 215, ISSN: 1746-8094.

Halford, J. J., Schalkoff, R. J., Zhou, J., Benbadis, S. R., Tatum, W. O., Turner, R. P., Sinha, S. R., Fountain, N. B., Arain, A., Pritchard, P. B., et al. (2013). Standardized database development for EEG epileptiform transient detection: EEGnet scoring system and machine learning analysis. Journal of Neuroscience Methods, 212(2):308-316.

Halford, J. J., Westover, M. B., LaRoche, S. M., Macken, M. P., Kutluay, E., Edwards, J. C., Bonilha, L., Kalamangalam, G. P., Ding, K., Hopp, J. L., et al. (2018). Interictal epileptiform discharge detection in EEG in different practice settings. Journal of Clinical Neurophysiology, 35(5):375.

Hesse, C. W. and James, C. J. (2006). Tracking epileptiform activity in the multichannel ictal EEG using spatially constrained independent component analysis. In 2005 IEEE Engineering in Medicine and Biology 27th Annual Conference, pages 2067-2070. IEEE.

Hunyadi, B., Camps, D., Sorber, L., Van Paesschen, W., De Vos, M., Van Huffel, S., and De Lathauwer, L. (2014). Block term decomposition for modelling epileptic seizures. EURASIP Journal on Advances in Signal Processing, 2014(1):1-19.

Hunyadi, B., Dupont, P., Van Paesschen, W., and Van Huffel, S. (2017). Tensor decompositions and data fusion in epileptic electroencephalography and functional magnetic resonance imaging data. Wiley Interdisciplinary Reviews: Data Mining and Knowledge Discovery, 7(1):e1197.

Iasemidis, L. D., Sackellares, J. C., Zaveri, H. P., and Williams, W. J. (1990). Phase space topography and the Lyapunov exponent of electrocorticograms in partial seizures. Brain Topography, $2(3): 187-201$.

Indiradevi, K., Elias, E., Sathidevi, P., Dinesh Nayak, S., and Radhakrishnan, K. (2008). A multilevel wavelet approach for automatic detection of epileptic spikes in the electroencephalogram. Computers in Biology and Medicine, 38(7):805-816, ISSN: 0010-4825. 
Janca, R., Jezdik, P., Cmejla, R., Tomasek, M., Worrell, G. A., Stead, M., Wagenaar, J., Jefferys, J. G., Krsek, P., Komarek, V., et al. (2015). Detection of interictal epileptiform discharges using signal envelope distribution modelling: application to epileptic and non-epileptic intracranial recordings. Brain Topography, 28(1):172-183.

Jarchi, D., Andreu-Perez, J., Kiani, M., Vysata, O., Kuchynka, J., Prochazka, A., and Sanei, S. (2020). Recognition of patient groups with sleep related disorders using bio-signal processing and deep learning. Sensors, 20(9):2594.

Jiang, Y., Chen, W., Zhang, T., Li, M., You, Y., and Zheng, X. (2020). Developing multi-component dictionary-based sparse representation for automatic detection of epileptic EEG spikes. Biomedical Signal Processing and Control, 60:101966, ISSN: 1746-8094.

Johansen, A. R., Jin, J., Maszczyk, T., Dauwels, J., Cash, S. S., and Westover, M. B. (2016). Epileptiform spike detection via convolutional neural networks. In 2016 IEEE International Conference on Acoustics, Speech and Signal Processing (ICASSP), pages 754-758. IEEE.

Kane, N., Acharya, J., Beniczky, S., Caboclo, L., Finnigan, S., Kaplan, P. W., Shibasaki, H., Pressler, R., and van Putten, M. J. (2017). A revised glossary of terms most commonly used by clinical electroencephalographers and updated proposal for the report format of the EEG findings. Revision 2017. Clinical Neurophysiology Practice, 2:170.

Kobayashi, K., James, C., Nakahori, T., Akiyama, T., and Gotman, J. (1999). Isolation of epileptiform discharges from unaveraged EEG by independent component analysis. Clinical Neurophysiology, 110(10):1755 - 1763, ISSN: 1388-2457.

Kobayashi, K., Merlet, I., and Gotman, J. (2001). Separation of spikes from background by independent component analysis with dipole modeling and comparison to intracranial recording. Clinical Neurophysiology, 112(3):405 - 413, ISSN: 1388-2457.

Kolda, T. G. (2006). Multilinear operators for higher-order decompositions. Technical report, Sandia National Laboratories.

Kolda, T. G. and Bader, B. W. (2009). Tensor decompositions and applications. SIAM Review, 51(3):455-500.

Le, T. X., Le, T. T., Dinh, V. V., Tran, Q. L., Nguyen, L. T., and Nguyen, D. T. (2018). Deep learning for epileptic spike detection. VNU Journal of Science: Computer Science and Communication Engineering, 33(2):1-13.

Lodder, S. S., Askamp, J., and van Putten, M. J. (2013). Inter-ictal spike detection using a database of smart templates. Clinical Neurophysiology, 124(12):2328-2335. 
Lodder, S. S. and van Putten, M. J. (2014). A self-adapting system for the automated detection of inter-ictal epileptiform discharges. PloS one, 9(1):e85180.

Medvedev, A. (2001). Temporal binding at gamma frequencies in the brain: Paving the way to epilepsy? Australasian Physics \& Engineering Sciences in Medicine, 24(1):37-48.

Medvedev, A., Agoureeva, G., and Murro, A. (2019). A long short-term memory neural network for the detection of epileptiform spikes and high frequency oscillations. Scientific Reports, 9(1):110.

Mirowski, P. W., LeCun, Y., Madhavan, D., and Kuzniecky, R. (2008). Comparing SVM and convolutional networks for epileptic seizure prediction from intracranial EEG. In 2008 IEEE Workshop on Machine Learning for Signal Processing, pages 244-249. IEEE.

Nayak, D., Valentin, A., Alarcon, G., Seoane, J. J. G., Brunnhuber, F., Juler, J., Polkey, C. E., and Binnie, C. D. (2004). Characteristics of scalp electrical fields associated with deep medial temporal epileptiform discharges. Clinical Neurophysiology, 115(6):1423-1435.

Ontivero-Ortega, M., Garcia-Puente, Y., and Martínez-Montes, E. (2015). Comparison of classifiers to detect epileptic seizures via PARAFAC decomposition. In VI Latin American Congress on Biomedical Engineering CLAIB 2014, Paraná, Argentina 29, 308331 October 2014, pages 500-503. Springer.

Özdamar, Ö. and Kalayci, T. (1998). Detection of spikes with artificial neural networks using raw EEG. Computers and Biomedical Research, 31(2):122-142.

Polat, K. and Güneş, S. (2007). Classification of epileptiform EEG using a hybrid system based on decision tree classifier and fast Fourier transform. Applied Mathematics and Computation, 187(2):1017 - 1026, ISSN: 0096-3003.

Prasanth, T., Thomas, J., Yuvaraj, R., Jing, J., Cash, S. S., Chaudhari, R., Leng, T. Y., Rathakrishnan, R., Rohit, S., Saini, V., et al. (2020). Deep learning for interictal epileptiform spike detection from scalp EEG frequency sub bands. In 2020 42nd Annual International Conference of the IEEE Engineering in Medicine $\mathcal{E}$ Biology Society (EMBC), pages 3703-3706. IEEE.

Procházka, A., Charvátová, H., Vyšata, O., Jarchi, D., and Sanei, S. (2020). Discrimination of cycling patterns using accelerometric data and deep learning techniques. Neural Computing and Applications, pages 1-11.

Sanei, S. (2013). Adaptive Processing of Brain Signals. John Wiley \& Sons. 
Sartoretto, F. and Ermani, M. (1999). Automatic detection of epileptiform activity by single-level wavelet analysis. Clinical Neurophysiology, 110(2):239 - 249, ISSN: 1388-2457.

Shi, X., Chen, Z., Wang, H., Yeung, D., Wong, W., and Woo, W. (2015). Convolutional LSTM network: A machine learning approach for precipitation nowcasting.

Spyrou, L., Kouchaki, S., and Sanei, S. (2015). Multiview classification of brain data through tensor factorisation. In 2015 IEEE 25th International Workshop on Machine Learning for Signal Processing (MLSP), pages 1-6. IEEE.

Spyrou, L., Kouchaki, S., and Sanei, S. (2018). Multiview classification and dimensionality reduction of scalp and intracranial EEG data through tensor factorisation. Journal of Signal Processing Systems, 90(2):273-284.

Spyrou, L., Martín-Lopez, D., Valentín, A., Alarcón, G., and Sanei, S. (2016). Detection of intracranial signatures of interictal epileptiform discharges from concurrent scalp EEG. International Journal of Neural Systems, 26(04):1650016.

Spyrou, L. and Sanei, S. (2016). Coupled dictionary learning for multimodal data: An application to concurrent intracranial and scalp EEG. In 2016 IEEE International Conference on Acoustics, Speech and Signal Processing (ICASSP), pages 2349-2353. IEEE.

Stevens, J. R., Lonsbury, B. L., and Goel, S. L. (1972). Seizure occurrence and interspike interval: Telemetered electroencephalogram studies. Archives of Neurology, 26(5):409-419.

Su, J., Byeon, W., Kossaifi, J., Huang, F., Kautz, J., and Anandkumar, A. (2020). Convolutional tensor-train LSTM for spatio-temporal learning.

Thanh, L. T., Dao, N. T. A., Dung, N. V., Trung, N. L., and Abed-Meraim, K. (2020). Multi-channel EEG epileptic spike detection by a new method of tensor decomposition. Journal of Neural Engineering, 17(1):016023.

Thomas, J., Jin, J., Dauwels, J., Cash, S. S., and Westover, M. B. (2017). Automated epileptiform spike detection via affinity propagation-based template matching. In 2017 39th Annual International Conference of the IEEE Engineering in Medicine and Biology Society (EMBC), pages 3057-3060. IEEE.

Thomas, J., Jin, J., Thangavel, P., Bagheri, E., Yuvaraj, R., Dauwels, J., Rathakrishnan, R., Halford, J. J., Cash, S. S., and Westover, B. (2020). Automated detection of interictal epileptiform discharges from scalp electroencephalograms by convolutional neural networks. International Journal of Neural Systems, pages 2050030-2050030. 
Tjepkema-Cloostermans, M. C., de Carvalho, R. C., and van Putten, M. J. (2018). Deep learning for detection of focal epileptiform discharges from scalp EEG recordings. Clinical Neurophysiology, 129(10):2191-2196.

Torre, J. F., Alarcon, G., Binnie, C., and Polkey, C. (1999). Comparison of sphenoidal, foramen ovale and anterior temporal placements for detecting interictal epileptiform discharges in presurgical assessment for temporal lobe epilepsy. Clinical Neurophysiology, 110(5):895-904.

Tzallas, A., Karvelis, P., Katsis, C., Fotiadis, D., Giannopoulos, S., and Konitsiotis, S. (2006). A method for classification of transient events in EEG recordings: Application to epilepsy diagnosis. Methods of Information in Medicine, 45(06):610-621.

Tzallas, A. T., Tsipouras, M. G., Tsalikakis, D. G., Karvounis, E. C., Astrakas, L., Konitsiotis, S., and Tzaphlidou, M. (2012). Automated epileptic seizure detection methods: A review study. Epilepsy - Histological, Electroencephalographic and Psychological Aspects, pages 75-98.

Wang, C., Zou, J., Zhang, J., Wang, M., and Wang, R. (2010). Feature extraction and recognition of epileptiform activity in EEG by combining PCA with ApEn. Cognitive Neurodynamics, 4(3):233-240.

Wang, Z., Wu, D., Dong, F., Cao, J., Jiang, T., and Liu, J. (2020). A novel spike detection algorithm based on multi-channel of BECT EEG signals. IEEE Transactions on Circuits and Systems II: Express Briefs, 67(12):3592-3596.

Webber, W., Litt, B., Wilson, K., and Lesser, R. P. (1994). Practical detection of epileptiform discharges (EDs) in the EEG using an artificial neural network: A comparison of raw and parameterized EEG data. Electroencephalography and Clinical Neurophysiology, 91(3):194-204.

Webber, W. R. S., Litt, B., Lesser, R., Fisher, R., and Bankman, I. (1993). Automatic EEG spike detection: What should the computer imitate? Electroencephalography and Clinical Neurophysiology, 87(6):364-373.

Wei, B., Zhao, X., Shi, L., Xu, L., Liu, T., and Zhang, J. (2021). A deep learning framework with multi-perspective fusion for interictal epileptiform discharges detection in scalp electroencephalogram. Journal of Neural Engineering, http://iopscience.iop.org/article/10. 1088/1741-2552/ac0d60.

Wieser, H., Elger, C., and Stodieck, S. (1985). The 'foramen ovale electrode': a new recording method for the preoperative evaluation of patients suffering from mesio-basal temporal lobe epilepsy. Electroencephalography and Clinical Neurophysiology, 61(4):314-322. 
Yamazaki, M., Tucker, D. M., Fujimoto, A., Yamazoe, T., Okanishi, T., Yokota, T., Enoki, H., and Yamamoto, T. (2012). Comparison of dense array EEG with simultaneous intracranial EEG for interictal spike detection and localization. Epilepsy Research, 98(2-3):166-173.

Yuan, Q., Zhou, W., Yuan, S., Li, X., Wang, J., and Jia, G. (2014). Epileptic EEG classification based on kernel sparse representation. International Journal of Neural Systems, 24(04):1450015.

Yuan, S., Liu, J., Shang, J., Xu, F., Dai, L., and Kong, X. (2020). Automatic seizure prediction based on modified Stockwell transform and tensor decomposition. In 2020 IEEE International Conference on Bioinformatics and Biomedicine (BIBM), pages 1503-1509. IEEE.

Yuan, S., Zhou, W., Wu, Q., and Zhang, Y. (2016). Epileptic seizure detection with Log-Euclidean gaussian kernel-based sparse representation. International journal of Neural Systems, 26(03):1650011.

Zhu, J., Lin, C., Chang, S., Wang, J., Peng, T., and Chien, Y. (2015). Analysis of spike waves in epilepsy using Hilbert-Huang transform. Journal of Medical Systems, 39(1):1-13. 


\section{Figure 1}

Lateral and Basal X-radiographs Showing Scalp and Intracranial Foramen Ovale (FO) Electrodes. Red Arrows Point to FO Electrods (Spyrou et al., 2016).

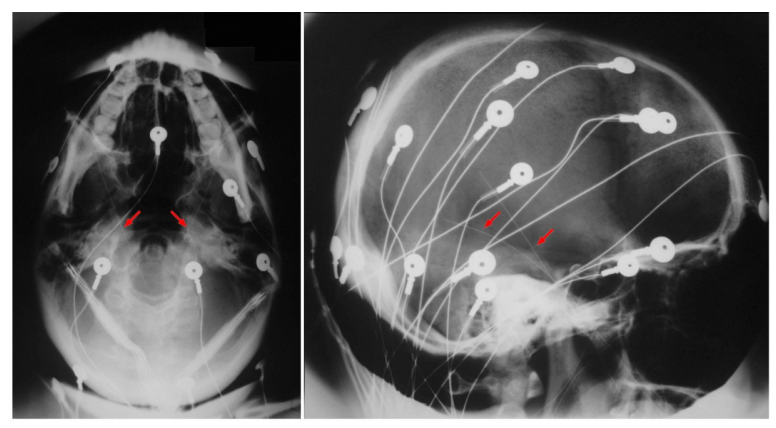


Figure 2

CANDECOMP/PARAFAC Decomposition of a Three-way Array.

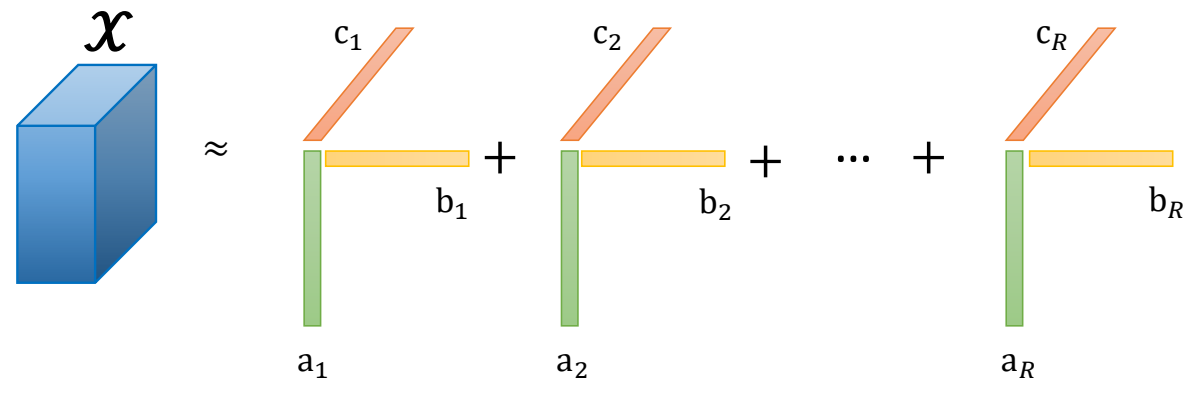


Figure 3

Tucker Decomposition of a Three-way Array.

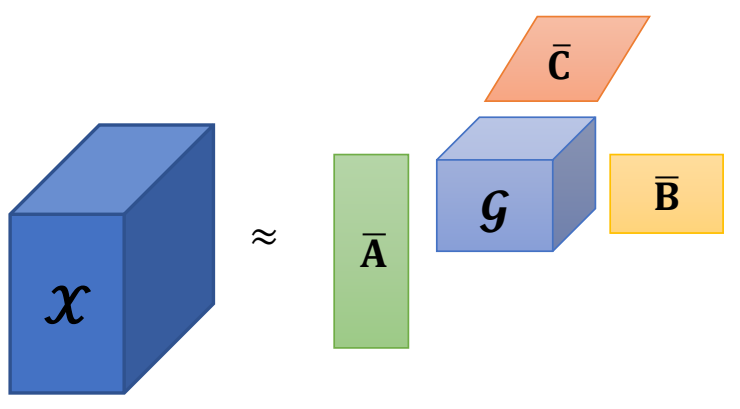




\section{Figure 4}

The ED Detection System Proposed for the Subject-dependent Classification Approach Adopted from Abdi-Sargezeh et al. (2021b). $\mathcal{X}$ Includes the ED Segments only, N. CPD Is Applied to $\mathcal{X}$ to Decompose it to Temporal, Spatial, and Segmental Factors. $\mathbf{X}_{k}(k=1, \ldots, K)$ Is an ED or Non-ED Segment from the Training or Test Data Which Is Projected onto the Spatial Components $\mathbf{B} . \mathbf{Y}_{k}$ Represents the Same Segment after Projection.

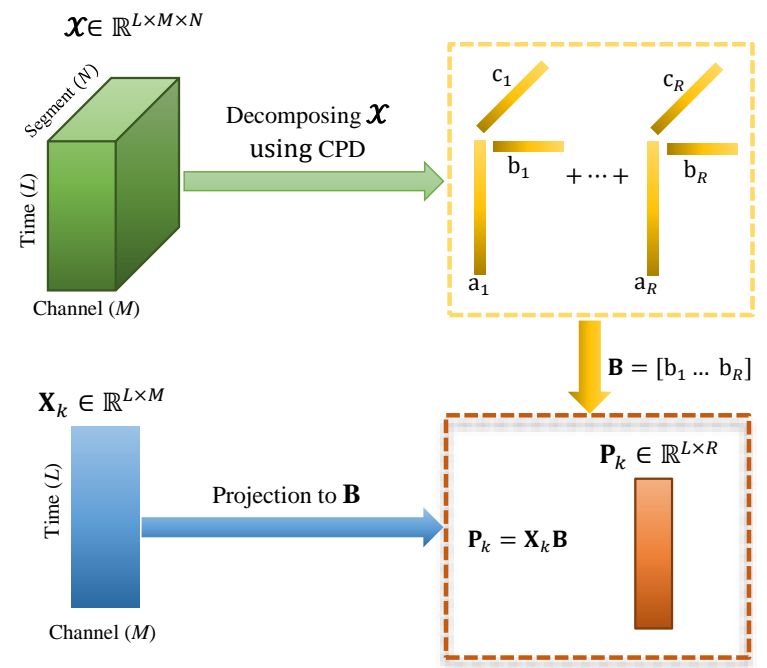


Figure 5

Representation of a 4-way Tensor (Spyrou et al., 2015).

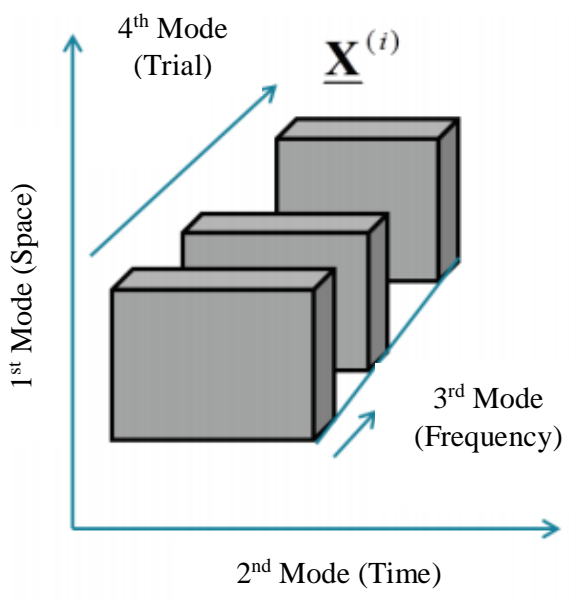




\section{Figure 6}

Schematic Diagram of a Basic Convolutional Neural Network Architecture.

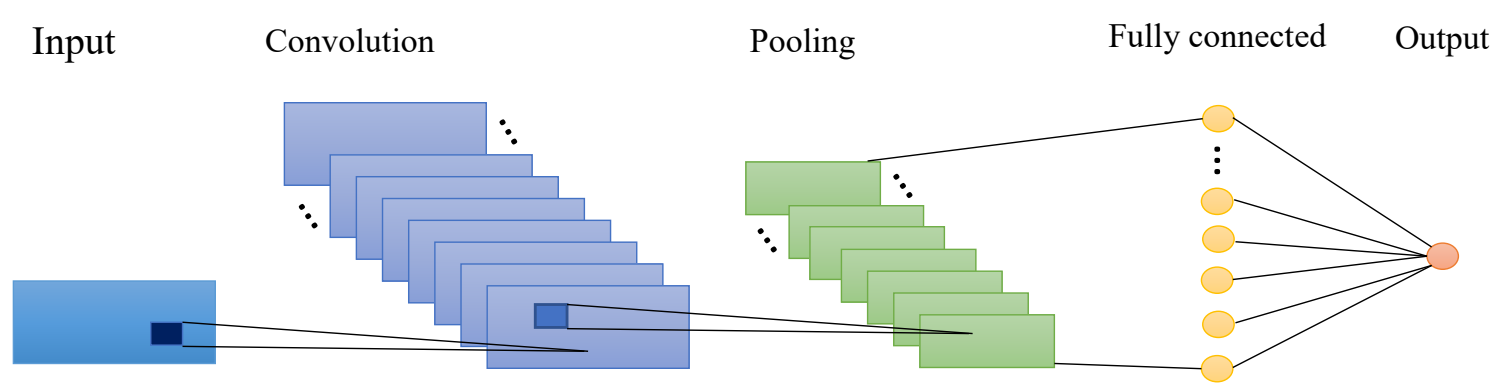


Figure 7

Samples of Epileptiform Discharge (ED) Waveforms with Scores 1 to 5 and a Non-ED Waveform. (a) The non-ED Segment, and (b)-(f) Respectively the ED Segments with Scores 1 to 5 for Lowest (Highest Uncertainty) to Highest (Lowest Uncertainty) Probability Respectively. Channels R1 to R6 and L1 to L6 Correspond to Intracranial EEG Recordings. The EDs Start at 160ms. In the Figure, IED Is the Written Abbreviation of Interictal Epileptiform Discharges. The Figure Is Adopted from Abdi-Sargezeh et al. (2021b).

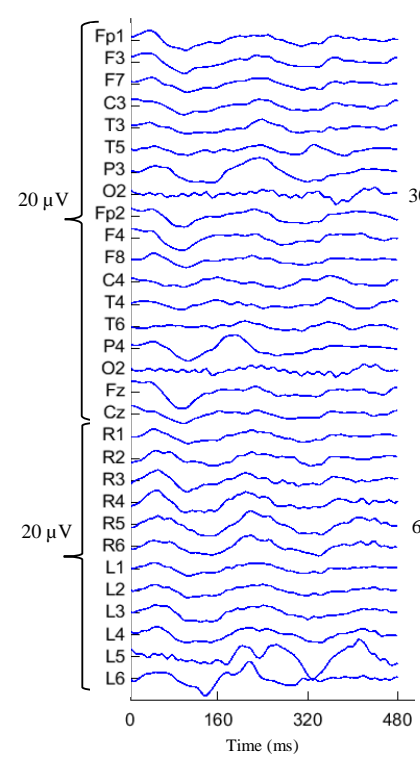

(a) Non-IED

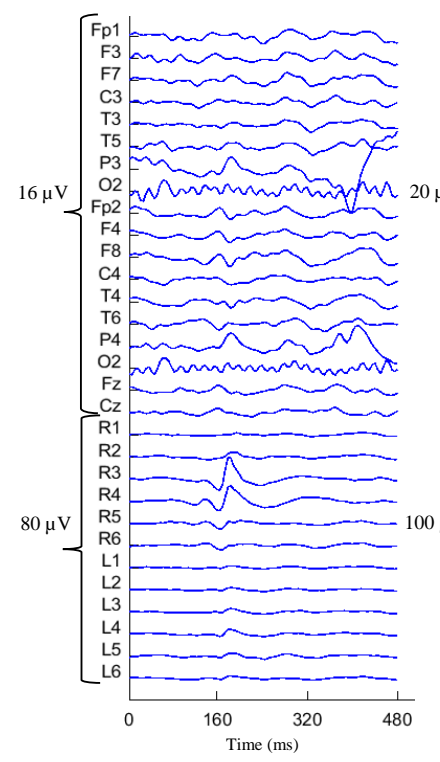

(d) Score 3 IED

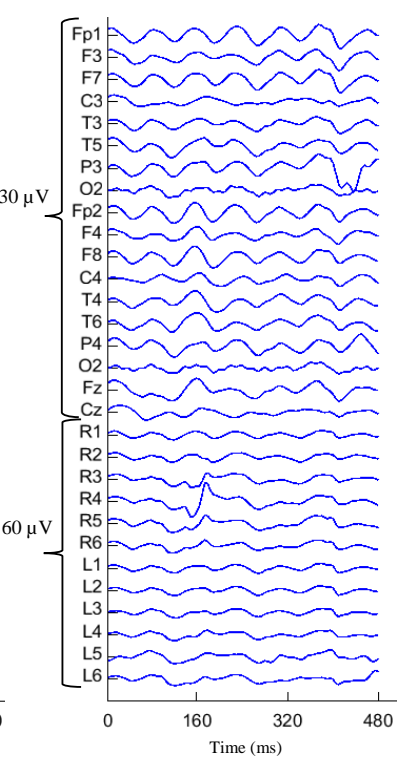

(b) Score 1 IED

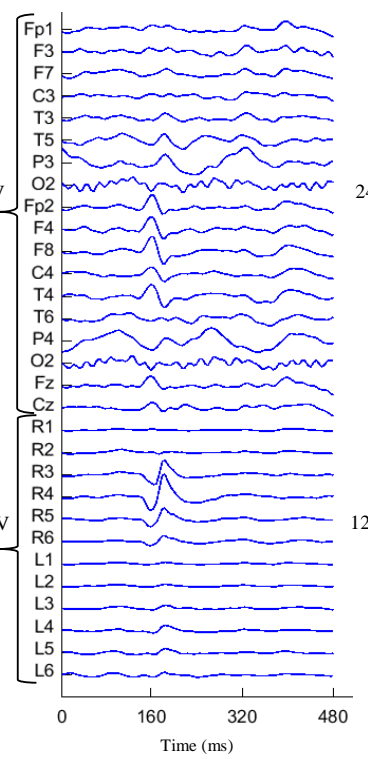

(e) Score 4 IED

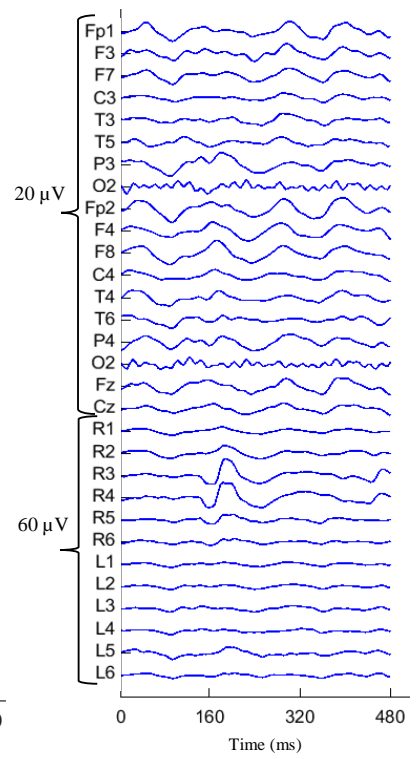

(c) Score 2 IED

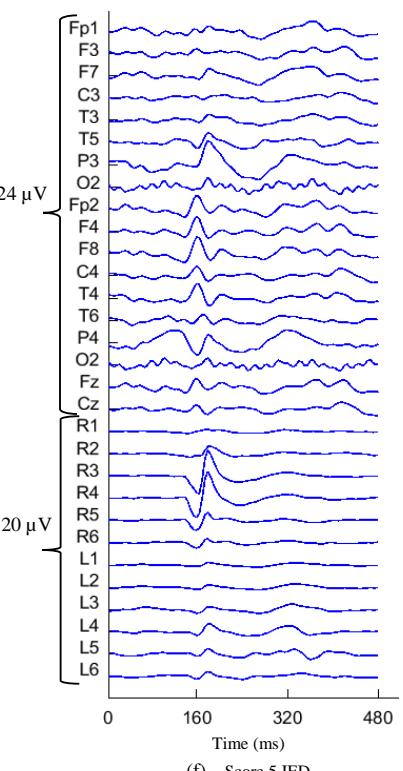

(f) Score 5 IED 


\section{Figure 8}

Topology of Asymmetric-Symmetric Autoencoder Network. x Is the scalp EEG (sEEG), $\mathbf{y}_{1}$ Is the Hidden Layer of the Asymmetric Autoencoder (AAE), $\mathbf{z}_{1}$ and $\mathbf{z}_{1}$ Are the Estimated Sources of intracranial EEG (iEEG), $\mathbf{y}_{2}$ Is the Hidden Layer of the Autoencoder $(A E), \mathbf{W}^{e}$ and $\mathbf{W}^{d}$ Are the Weights of the $A A E$ and $W$ Are the Tied Weights of the AE (Antoniades et al., 2018).

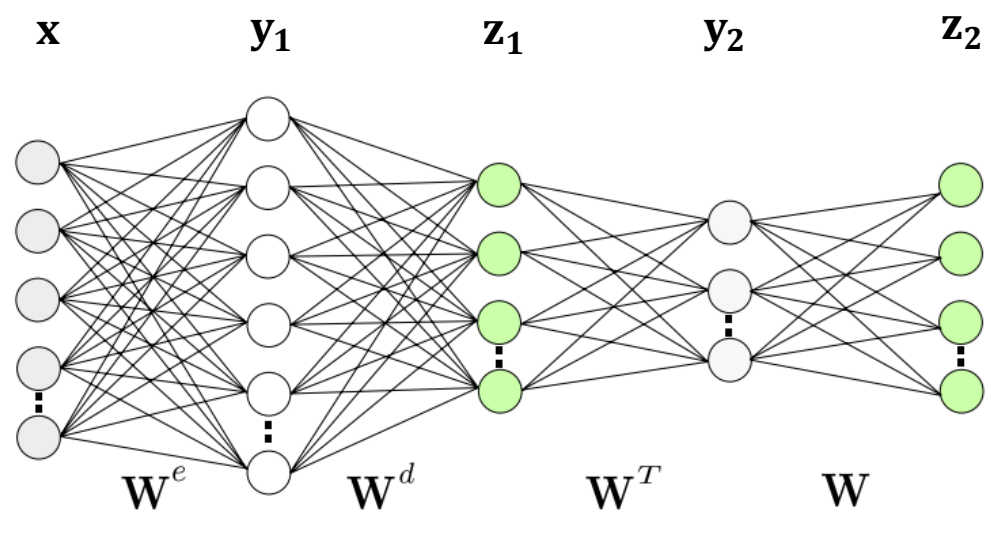

AAE to Map sEEG to iEEG

Symmetric AE to

Enhance Pseudo-iEEG 


\section{Figure 9}

Estimation of Intracranial EEG (iEEG) from the Scalp Epileptiform Discharges (EDs) and Non-ED Segments (Averaged Over All Channels) Using Asymmetric Autoencoder (AAE) and AsymmetricSymmetric Autoencoder (ASAE) (Antoniades et al., 2018). In the Case of ASAE, the Additional Symmetric Layer Led to a Smoother Estimation of the Intracranial Data. The ED Started from the $32^{\text {nd }}$ Sample.
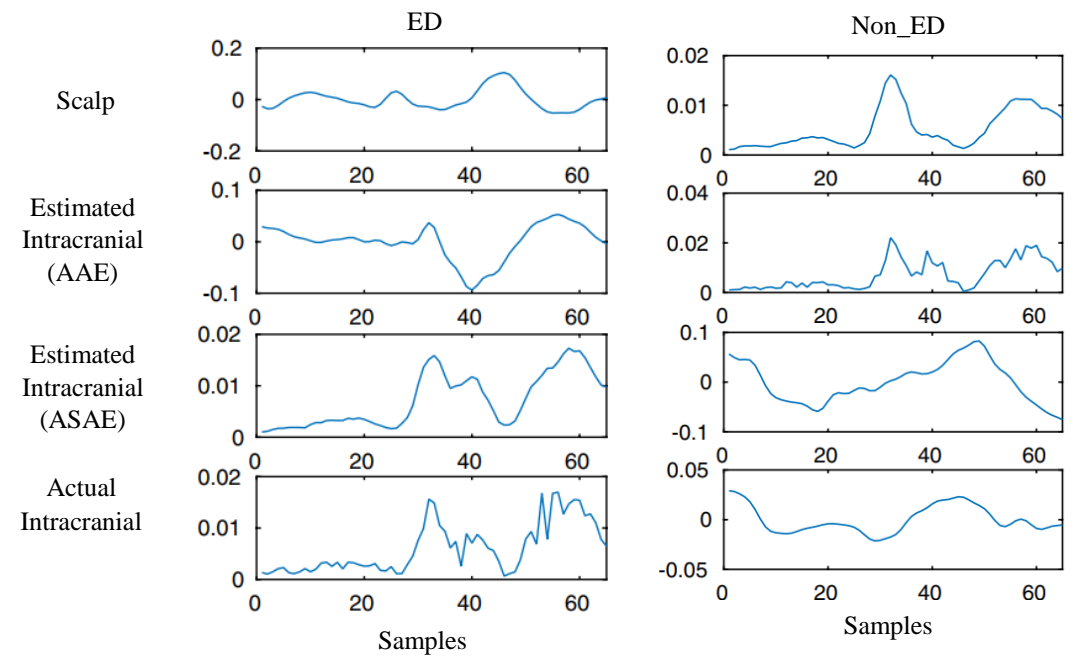
Table 1

Some Datasets Used in Epileptiform Discharge (ED) Detection Studies.

\begin{tabular}{|c|c|c|c|c|}
\hline Study & Recording & $\begin{array}{l}\text { Num. of } \\
\text { channels }\end{array}$ & $\begin{array}{l}\text { Num. of } \\
\text { subjects }\end{array}$ & Num. of EDs/spikes \\
\hline Indiradevi et al. 2008 & sEEG & 18 & 22 & 684 \\
\hline$\overline{\text { Le et al. }} \overline{2018}$ & sEEG & 32 & 19 & 1491 \\
\hline \begin{tabular}{|l|l} 
Lodder et al. 2013 & Lodder and \\
\end{tabular} & sEEG & - & 23 & 723 \\
\hline van Putten 2014 & & & & \\
\hline Wang et al. $\overline{2020}$ & sEEG & 21 & 7 & 4850 \\
\hline Thanh et al. 2020 & sEEG & 19 & 7 & 1442 \\
\hline Abou Jaoude et al. 2020 & iEEG & 4 & 46 & 13959 \\
\hline Johansen et al. 2016 & sEEG & 19 & 5 & 7500 \\
\hline $\begin{array}{l}\text { Thomas et al. } 2017,2020 \\
\text { anth et al. } 2020\end{array}$ & sEEG & 19 & $50 / 545$ & $8929 / 14170$ \\
\hline \begin{tabular}{|l|l|} 
Fukumori et al. & 2019 \\
\end{tabular} & sEEG & - & $5 / 50$ & $2897 / 15899 / 16008$ \\
\hline \begin{tabular}{|lll} 
Janca et al. 2015 & Geng et al. & 2021 \\
\end{tabular} & iEEG & 15 & 7 & 1956 \\
\hline $\begin{array}{l}\text { Spyrou et al. } 2015, \mid 2016,2018, \\
\text { Antoniades et al. } 2016,2017,2018 \\
\text { Abdi-Sargezeh et al. } \mid 2021 \mathrm{~b} \text { c }\end{array}$ & $\begin{array}{l}\text { Concurrent } \\
\text { sEEG and } \\
\text { iEEG }\end{array}$ & $\begin{array}{l}20 \text { scalp and } \\
12 \text { FO elec- } \\
\text { trodes }\end{array}$ & $25 / 24 / 18$ & $7831 / 6609 / 2776$ \\
\hline
\end{tabular}


Table 2

Summary of Patients' information Used in the Public Intracranial Epileptiform Discharges Dataset, Adopted from Janca et al. (2015).

\begin{tabular}{llllll}
\hline Patient & Age (sex) & Num. of GS & Epilepsy location & $\begin{array}{l}\text { Seizure duration } \\
\text { (years) }\end{array}$ & Pathology \\
\hline 1 & $24(\mathrm{~F})$ & 613 & Right frontal & 6 & No abnormality \\
\hline 2 & $37(\mathrm{~F})$ & 453 & Left frontal & 28 & No abnormality \\
\hline 3 & $17(\mathrm{~F})$ & 21 & Left temporal & 2 & FCD Ib \\
\hline 4 & $8(\mathrm{~F})$ & 3 & Right frontal & 3 & FCD IIb \\
\hline 5 & $14(\mathrm{~F})$ & 319 & Right multilobar & 8 & HS, FCD Ia \\
\hline 6 & $31(\mathrm{M})$ & 335 & Right frontal & 27 & FCD IIb \\
\hline 7 & $10(\mathrm{M})$ & 212 & Left multilobar & 3 & FCD IIa \\
\hline$* * *$ M: Male; F: Female; GS: Golden Standard Spikes; FCD: Focal Cortical Dysplasia; HS: Hippocampal Sclerosis.
\end{tabular}


Table 3

Studies Employing Multi-way Analysis Methods for Seizure or Epileptiform Discharge (ED) Detection.

\begin{tabular}{|c|c|c|c|c|c|c|}
\hline Study & Application & Recording & Tensorization & Decomposition & $\begin{array}{l}\text { Features for classifica- } \\
\text { tion }\end{array}$ & Performance \\
\hline $\begin{array}{l}\text { Acar et al. } \\
2007 \mathrm{~b}\end{array}$ & $\begin{array}{l}\text { Seizure } \\
\text { detection }\end{array}$ & sEEG & CWT & N-PLS & $\begin{array}{l}\text { The component ma- } \\
\text { trices extracted from } \\
\text { N-PLS model }\end{array}$ & $\begin{array}{l}\mathrm{ACC}=77 \%-96 \% \text { for dif- } \\
\text { ferent subjects }\end{array}$ \\
\hline \begin{tabular}{|l|} 
Spyrou \\
et al. 2015 \\
\end{tabular} & $\begin{array}{l}\mathrm{ED} \\
\text { detection }\end{array}$ & iEEG & Spectrogram & TD & $\begin{array}{l}\text { Spatial factor matrix } \\
\text { extracted from TD }\end{array}$ & $\mathrm{ACC}=86 \%$ \\
\hline \begin{tabular}{|l|} 
Spyrou \\
et al. 2018
\end{tabular} & $\begin{array}{l}\text { ED } \\
\text { detection }\end{array}$ & sEEG & Spectrogram & TD & $\begin{array}{l}\text { Factor matrices and the } \\
\text { core tensor extracted } \\
\text { from TD }\end{array}$ & $\begin{array}{l}\text { Different performance was } \\
\text { obtained by projecting the } \\
\text { test data onto the different } \\
\text { factors }\end{array}$ \\
\hline \begin{tabular}{|l|} 
Aldana \\
et al. 2018
\end{tabular} & $\begin{array}{l}\text { Seizure } \\
\text { detection }\end{array}$ & sEEG & CWT/HHT & CPD/BTD & $\begin{array}{l}\text { Factor matrices ex- } \\
\text { tracted from CPD or } \\
\text { BTD }\end{array}$ & $\begin{array}{l}\mathrm{ACC}=\mathrm{SEN}=\mathrm{SPC}= \\
99 \%\end{array}$ \\
\hline $\begin{array}{l}\text { Yuan et al. } \\
2020\end{array}$ & $\begin{array}{l}\text { Seizure } \\
\text { prediction }\end{array}$ & iEEG & $\begin{array}{l}\text { Stockwell } \\
\text { transform }\end{array}$ & TD & $\begin{array}{l}\text { Core tensor extracted } \\
\text { from TD }\end{array}$ & $\begin{array}{l}\text { SEN }=88 \% \text { for the seizure } \\
\text { occurrence period of } 30 \\
\text { min; SEN }=97 \% \text { for the } \\
\text { seizure occurrence period } \\
\text { of } 50 \mathrm{~min}\end{array}$ \\
\hline \begin{tabular}{|l|} 
Thanh \\
et al. 2020
\end{tabular} & $\begin{array}{l}\text { Epileptiform } \\
\text { spike } \\
\text { detection }\end{array}$ & sEEG & CWT & NTD & $\begin{array}{l}\text { Projecting spikes and } \\
\text { non-spikes into factor } \\
\text { matrices extracted from } \\
\text { NTD }\end{array}$ & $\begin{array}{l}\mathrm{ACC}=90 \%, \mathrm{SEN}=80 \% \\
\mathrm{SPC}=90 \%\end{array}$ \\
\hline \begin{tabular}{|l|} 
Abdi- \\
Sargezeh \\
et al. \\
$2021 b$ \\
\end{tabular} & $\begin{array}{l}\text { ED } \\
\text { detection }\end{array}$ & sEEG & ED segment & CPD & $\begin{array}{l}\text { Projecting ED and } \\
\text { non-ED segments into } \\
\text { spatial factors extracted } \\
\text { from CPD }\end{array}$ & $\begin{array}{l}\mathrm{ACC}=80 \%, \mathrm{SEN}=77 \% \\
\mathrm{SPC}=82 \%\end{array}$ \\
\hline \begin{tabular}{|l|} 
Abdi- \\
Sargezeh \\
et al. \\
$2021 \mathrm{c}$ \\
\end{tabular} & $\begin{array}{l}\text { ED } \\
\text { detection }\end{array}$ & sEEG & ED segment & CPD & $\begin{array}{l}\text { Projecting ED and } \\
\text { non-ED segments into } \\
\text { spatial factors extracted } \\
\text { from CPD }\end{array}$ & $\begin{array}{l}\mathrm{ACC}=79 \%, \mathrm{SEN}=71 \% \\
\mathrm{SPC}=87 \%\end{array}$ \\
\hline
\end{tabular}


Table 4

Studies Employing Deep Learning Algorithms to Detect Epileptiform Discharges (EDs).

\begin{tabular}{|c|c|c|c|c|c|}
\hline Study & Application & Recording & Algorithm & $\begin{array}{l}\text { Input features of } \\
\text { algorithm }\end{array}$ & Performance \\
\hline \begin{tabular}{|l|} 
Antoniades \\
et al. 2016 \\
\end{tabular} & ED detection & iEEG & $\mathrm{CNN}$ & The spectrogram of iEEG & $\mathrm{ACC}=87 \%$ \\
\hline \begin{tabular}{|l|} 
Johansen \\
et al. 2016
\end{tabular} & $\begin{array}{l}\text { Epileptiform } \\
\text { spike detection }\end{array}$ & sEEG & $\mathrm{CNN}$ & Raw EEG & $\mathrm{AUC}=0.94$ \\
\hline \begin{tabular}{|l|} 
Antoniades \\
et al. 2017
\end{tabular} & $\begin{array}{l}\text { Multiclass ED } \\
\text { detection }\end{array}$ & iEEG & $\mathrm{CNN}$ & Raw EEG & $\mathrm{ACC}=89 \%$ \\
\hline \begin{tabular}{|l|} 
Antoniades \\
et al. 2018
\end{tabular} & ED detection & $\begin{array}{l}\text { Concurrent } \\
\text { sEEG and } \\
\text { iEEG }\end{array}$ & ASAE and CNN & $\begin{array}{l}\text { Estimated iEEG from } \\
\text { sEEG }\end{array}$ & $\mathrm{ACC}=68 \%$ \\
\hline \begin{tabular}{|l|} 
Tjepkema- \\
Cloostermans \\
et al. 2018 \\
\end{tabular} & ED detection & sEEG & $\begin{array}{l}\text { CNN, LSTM, } \\
\text { stacked CNN and } \\
\text { LSTM }\end{array}$ & Raw EEG & $\mathrm{SEN}=47 \%, \mathrm{SPC}=98 \%$ \\
\hline \begin{tabular}{|l|} 
Le et \\
2018 \\
\end{tabular} & $\begin{array}{l}\text { Epileptiform } \\
\text { spike detection }\end{array}$ & sEEG & $\mathrm{DBN}$ & Wavelet features & $\begin{array}{l}\mathrm{ACC}=87 \%, \mathrm{SPC}=97 \%, \\
\mathrm{AUC}=0.95\end{array}$ \\
\hline \begin{tabular}{|l} 
Medvedev \\
et al. 2019
\end{tabular} & $\begin{array}{l}\text { Spike and rip- } \\
\text { ple detection }\end{array}$ & iEEG & LSTM & $\begin{array}{l}\text { Power spectrogram of } \\
\text { sub-bands from } 4 \text { to } 236 \\
\mathrm{~Hz}\end{array}$ & $\mathrm{ACC}>90 \%$ for all subjects \\
\hline \begin{tabular}{|l|} 
Fukumori \\
et al. 2019
\end{tabular} & $\begin{array}{l}\text { Epileptiform } \\
\text { spike detection }\end{array}$ & sEEG & $\begin{array}{l}\text { CNN, LSTM, } \\
\text { GRU }\end{array}$ & $\begin{array}{l}\text { Frequency sub-bands ob- } \\
\text { tained using DWT }\end{array}$ & $\begin{array}{l}0.49<\mathrm{AUC}<0.96 \text { for differ- } \\
\text { ent subjects and models }\end{array}$ \\
\hline $\begin{array}{l}\text { Abou Jaou } \\
\text { et al. } 2020 \\
\end{array}$ & ED detection & iEEG & $1 \mathrm{D} \mathrm{CNN}$ & A single bipolar channel & $\begin{array}{l}0.49<\text { AUC }<0.96 \text { for differ- } \\
\text { ent subjects and models }\end{array}$ \\
\hline $\begin{array}{l}\text { Thomas } \\
\text { et al. } 2020\end{array}$ & ED detection & sEEG & 1D CNN & Raw EEG & $\begin{array}{l}\mathrm{FP} / \mathrm{min}=0.2 \text { when } \\
\mathrm{SEN}=80 \%\end{array}$ \\
\hline $\begin{array}{l}\text { Prasanth } \\
\text { et al. } 2020\end{array}$ & ED detection & sEEG & CNN & $\begin{array}{l}\text { Raw EEG and frequency } \\
\text { sub-bands }\end{array}$ & $\begin{array}{l}\mathrm{FP} / \mathrm{min}=0.23 \text { when } \\
\mathrm{SEN}=90 \%\end{array}$ \\
\hline $\begin{array}{l}\text { Fürbass } \\
\text { et al. } 2020\end{array}$ & ED detection & sEEG & Fast R-CNN & Raw EEG & $\begin{array}{l}\mathrm{ACC}=80 \%, \mathrm{SEN}=89 \% \\
\mathrm{SPC}=70 \%\end{array}$ \\
\hline $\begin{array}{l}\text { Fukumori } \\
\text { et al. } 2020 \\
\end{array}$ & $\begin{array}{l}\text { Epileptiform } \\
\text { spike detection }\end{array}$ & sEEG & $\begin{array}{l}\text { Linear phase } \\
\text { CNN }\end{array}$ & Raw EEG & AUC $>0.9$ in most subjects \\
\hline $\begin{array}{l}\text { Fukumori } \\
\text { et al. } 2021\end{array}$ & $\begin{array}{l}\text { Epileptiform } \\
\text { spike detection }\end{array}$ & sEEG & $\begin{array}{l}\text { LSTM based on } \\
\text { self-attention }\end{array}$ & Raw EEG & $\mathrm{ACC}=90 \%$ \\
\hline \begin{tabular}{|l} 
Wei et al. \\
2021 \\
\end{tabular} & ED detection & sEEG & $\begin{array}{l}\text { A framework } \\
\text { based on CNN } \\
\text { and GRU }\end{array}$ & Raw EEG & $\begin{array}{l}\mathrm{ACC}=95 \%, \mathrm{SEN}=93 \% \\
\mathrm{SPC}=96 \%, \mathrm{AUC}=0.97\end{array}$ \\
\hline \begin{tabular}{|l|} 
Geng et al. \\
2021
\end{tabular} & ED detection & iEEG & $\begin{array}{l}\text { LSTM and the } \\
\text { proposed AC- } \\
\text { GAN }\end{array}$ & Real and synthetic EEG & AUC $>0.93$, ACC $>93 \%$ \\
\hline
\end{tabular}

